\title{
Relative Difficulties Of Daily Living Tasks With Retinitis Pigmentosa
}

Keziah Latham PhD FCOptom ${ }^{1,2}$, Mohammad Baranian MSc $^{2,3}$, Matthew A. Timmis PhD ${ }^{2,3}$, Andy Fisher BA CLVT ${ }^{4}$, Shahina Pardhan PhD MCOptom².

1. Department of Vision \& Hearing Sciences, Anglia Ruskin University, UK

2. Vision \& Eye Research Unit, Postgraduate Medical Institute, Anglia Ruskin University, UK

3. Sports and Exercise Sciences Research Group, Anglia Ruskin University, UK

4. Focal Point UK, Bridgend, UK.

Address for correspondence:

Dr Keziah Latham

Department of Vision and Hearing Sciences, Anglia Ruskin University, East Road, Cambridge, CB1 1PT, UK.

Keziah.latham@anglia.ac.uk

Word count: 5331 words

Tables: 12

Figures: 0

Submitted: 3 June 2016

Resubmission: 16 September 2016

Accepted: 31 October 2016 


\section{Abstract \\ Purpose}

To determine the relative difficulty of activity of daily living tasks for people with Retinitis

Pigmentosa (RP).

Methods

Participants with RP ( $n=166)$ rated the difficulty of tasks $(n=43)$ underpinning the Dutch Activity Inventory goals of mobility indoors and outdoors, shopping and using public transport. Demographic characteristics were also determined. Responses were Rasch analysed to determine properties of the scale, derive unidimensional subscales, and consider differential item functioning (DIF).

\section{$\underline{\text { Results }}$}

Following removal of one ill-fitting item, the remaining 42 tasks formed a scale with reasonable Rasch parameters but poor unidimensionality. The most difficult tasks were orienting in poor and bright light both indoors and outdoors, and avoiding peripheral obstacles outdoors. Eight subscales were derived with unidimensional properties, each of which could be considered as requiring similar skills. DIF identified that tasks from the 'poor light and obstacles' subscale were more difficult for those younger than the median age, non-users of mobility aids, and those not registered or registered sight impaired. Tasks from the 'finding products' and 'public transport' subscales were more difficult for those older than the median age, with longer duration of visual loss, users of mobility aids, and those registered severely sight impaired.

\section{Conclusions}

The most difficult tasks for people with RP of orienting in poor light and avoiding peripheral obstacles are relatively more difficult for those not registered as 'severely sight impaired', but are less difficult for those who use mobility aids. Mobility aids (guide dog or cane) therefore do benefit users in their perceived ability in these particular tasks. The derived unidimensional subscales reorganises the tasks from those grouped together by goal (researcher driven) to those perceived as requiring similar skills by people with RP (patient driven), and can be used as an evidence base for orientation and mobility training protocols.

Keywords: Retinitis Pigmentosa, Activities of Daily Living, Rehabilitation, Visual impairment, Rasch analysis, Orientation and mobility, Mobility aids 
1 Retinitis Pigmentosa is a genetic retinal dystrophy ${ }^{1}$ that primarily impairs peripheral vision. ${ }^{2}$ The condition is progressive, with visual loss taking place over a number of years following the presentation of initial symptoms. ${ }^{1,2}$ Loss of peripheral vision leads to particular difficulty with mobility, ${ }^{3-8}$ but also leads to activity limitations in a range of other tasks such as reading ${ }^{7,9}$ and visual search. $^{3}$

To determine the rehabilitation needs of visually impaired people across a full range of activities of daily living, the Dutch Activity Inventory ${ }^{10-12}$ has been designed as an adaptive instrument structured in terms of the World Health Organisation's International Classification of Functioning, Disability and Health. ${ }^{13}$ Respondents are asked the importance and difficulty of 47 goals underpinning ten objectives (learning and applying knowledge, general tasks and demands, communication, mobility, self-care, domestic life, interpersonal interactions, major life areas, community social and civil life, and emotional health). In a full administration of the instrument, for each goal that is of some importance and at least some difficulty, respondents are then asked the difficulty of a number of tasks underpinning that specific goal.

We have recently demonstrated that at goal level, mobility is the most challenging domain within the Dutch Activity Inventory for people with Retinitis Pigmentosa, ${ }^{14}$ and set this within the context of other challenging domains and goals. The specific daily living goals with which greatest difficulty was expressed were mobility outdoors, shopping, physical activity and / or sport, mobility indoors, and using public transport. Whilst several of these goals underpin the mobility domain within the instrument, shopping is considered under domestic life, and physical activity under community, social and civic life, highlighting that the difficulties faced by those with Retinitis Pigmentosa extend beyond mobility goals.

The purpose of the present study was to further investigate the difficulty of tasks underpinning the most difficult goals for people with Retinitis Pigmentosa, in order to determine areas of particular difficulty to address in rehabilitation. Whilst the purpose of the present study is therefore not 
specifically to validate the Dutch Activity Inventory, the use of analysis techniques used in questionnaire validation allows consideration of what the instrument tells us about the difficulty of activities of daily living with Retinitis Pigmentosa.

\section{Methods}

\section{$\underline{\text { Participants }}$}

Participants were recruited through the charity Retinitis Pigmentosa Fighting Blindness by advertising the study at their annual conference, and through their newsletter and social media pages. Participants of a previous study ${ }^{14}$ who had given their consent to be contacted were also approached. Inclusion criteria for the study were a self-reported diagnosis of Retinitis Pigmentosa, and age of at least 18 years. The resulting sample of 166 people is not the same as reported in our previous study ${ }^{14}$, but is the same sample as reported in a further study. ${ }^{15}$

The study was undertaken using online questionnaires. Potential participants were given the web address at which the study could be completed, which was hosted via surveygizmo. Informed consent was obtained from all participants once the nature of the study had been explained, by checking a tick box on the web page. Participants could not proceed to the study until they had consented to take part. The tenets of the Declaration of Helsinki were observed. Ethical approval was obtained from the Anglia Ruskin University Faculty of Science and Technology Research Ethics

\section{Committee.}

\section{Demographic information}

Participants were asked to report their age, gender, duration of visual impairment, visual impairment registration status (not registered, registered as 'sight impaired', or registered as 'severely sight impaired'), and whether they used a mobility aid (cane and / or guide dog). In the United Kingdom, people can be registered as sight impaired with full visual field and visual acuity 

or better if there is a 'gross' field defect. Severely sight impaired registration is available to those with visual acuity $<3 / 60$ and full visual field, visual acuity between $3 / 60$ and $6 / 60$ with a 'significantly contracted' field of vision, or visual acuity of $>6 / 60$ with a 'severely contracted' field of vision. ${ }^{16}$

\section{Dutch Activity Inventory}

Participants were asked to rate the difficulty of four goals that were found to be of greatest difficulty within the Dutch Activity Inventory at goal level (difficulty with mobility outdoors, shopping, mobility indoors, and using public transport). ${ }^{14}$ For each goal that was applicable and of some difficulty, the difficulty of the tasks underpinning the goal were asked. Three of these four goals were from the mobility domain, and the other (shopping) was from the domestic life domain. Although 'physical activity and / or sport' was also in the 'top five' most difficult goals, it was not assessed further here because the underlying task questions had to take into account a variety of different sports and activities that would reduce the applicability of each question to a small number of participants. The tasks underlying each goal (total of 43) are outlined in Tables 1-4. Note that the task questions were asked in association with the relevant goal, so that where questions were similar they were considered with respect to the given goal (e.g. 'Get somewhere without getting too tired' appears with relation to mobility outdoors as question 9, and with relation to the use of public transport as question 30). For each task, participants responded on a 6 point Likert scale. 0 indicated that the task was not important or not applicable to the participant and was analysed as missing data. A score of 1 indicated that the task was impossible without help, 2 was extremely difficult, 3 was moderately difficult, 4 was slightly difficult and 5 was not difficult. 
The Dutch Activity Inventory task questions were Rasch analysed using Winsteps version 3.91.00 (winsteps.com). Rasch analysis ${ }^{17}$ is a probabilistic measurement model which allows the conversion of ordinal responses to interval data, ${ }^{18}$ allowing application of parametric statistics. It also allows comment on the relative difficulty of items, the functional ability of individuals, and the degree to which a set of questions represent a unidimensional construct. ${ }^{19}$

Person and item measures are produced in logits, or log odds units, which represent the likelihood of a person having the ability to achieve an item, or an item being achievable for a person. The average logit value for items is arbitrarily set to zero. Given the scoring system employed, with higher scores indicating less difficulty with a task, higher derived person measures indicated that an individual had greater perceived ability, and a higher item difficulty indicated that more ability was needed to achieve an item, and thus that the task was more difficult.

Rasch analysis was initially undertaken with a single Andrich rating scale of all the task items considered together. ${ }^{20}$ Item fit was considered, as an indication of whether items were responded to similarly by participants. Items with an infit or outfit greater than 2.0 meansquare were iteratively removed on the grounds that their inclusion had the potential to harm the scale. ${ }^{21}$ Remaining items with infit and outfit values between 0.5 and 1.5 meansquare were considered to be contributing usefully to the scale, and those with fits between 1.5 and 2.0 were considered not to damage the scale and were thus retained. ${ }^{21}$

For the remaining items, category functioning was examined, with the required outcome being that all categories were utilised in order of functional ability, with each category the most common response at some point on the functional scale. This means that as an individual's perceived ability increases, their probability of selecting a higher value category increases in an ordered fashion. Item and person separation and reliability were noted, which provide an indication of the instrument's ability to reliably order items in terms of their difficulty, and respondents in terms of their ability, respectively. For people, values of at least 2.0 for separation and 0.80 for reliability were expected, 
and for items the equivalent values were 3.0 and $0.90 .{ }^{22}$ The targeting of the scale indicated how well matched the function of the participants was to the difficulty of the items, and was expected to be within \pm 1 logit. ${ }^{23}$

To investigate unidimensionality, or the extent to which all items are addressing a single construct, beyond the indications given by the item fits, Rasch residual-based principal components analysis was considered. The variance in the data accounted for by the Rasch dimension was first considered, with at least $60 \%$ of variance explained by the primary measure considered to demonstrate reasonable overall unidimensionality in the instrument. ${ }^{23}$ The unexplained variance or residuals were then decomposed to look for patterns indicating a secondary dimension to the data rather than random noise. Contrasts found within the residuals after the primary model had been extracted with the strength of at least two items, i.e., an eigenvalue of at least 2.0, were considered as evidence that the instrument did not assess a strictly unidimensional construct, ${ }^{21}$ and that there may be subscales within the items that might usefully be separated into different scales.

To consider whether the overview scale could be separated into more unidimensional subscales, items loading more than 0.4 onto the first contrast were selected as contributing significantly to the contrast and considered separately. These items were Rasch analysed as a separate scale, and the parameters examined, including the contrasts. The process was repeated until a stable unidimensional Rasch subscale was produced. If the parameters of this subscale were largely acceptable (as defined by the parameters considered for the overview scale, plus a first contrast of $<2$ eigenunits), the items were retained as a separate subscale. If the subscale produced was inadequate, the items were discarded. The process was then repeated for all the remaining items in the overview scale, excluding those items already considered in subscales, until no further significant contrasts (>2 eigenunits) remained in the data.

Differential item functioning was used to consider whether the items within the overview scale were of similar difficulty for all respondents, or were of particular difficulty for certain groups. Significant 
differential item functioning was considered as a contrast (difference between item difficulties for each group) of at least 0.50 logits and a significance of this difference of at least $p<.01$. A relatively conservative significance value was used, given the number of comparisons considered. Differential item functioning was considered for the demographic characteristics of age, duration of visual loss (both categorised by a median split of the data), gender (male v female), mobility aid usage (dog and / or cane used $v$ no aid used), and registration status (less severe loss (not registered and registered sight impaired) v more severe loss (severely sight impaired)).

\section{Results}

\section{Participants}

One hundred and sixty six people took part in the study. There were 91 females and 75 males, with a mean age of $50 \pm 16$ years (median 51.5 years, range $18-83$ years), and a mean duration of visual loss of $22 \pm 16$ years (median 16 years, range 6 months -70 years). Seventeen were not registered as visually impaired, 63 were 'sight impaired' and 86 were 'severely sight impaired'. Eighty four people used mobility aids (cane, dog or both) and 82 did not.

\section{Overview analysis of difficult tasks for people with Retinitis Pigmentosa}

One hundred and fifty nine participants expressed some difficulty with at least one of the four goals and were asked the relevant task questions. Mobility outdoors was of importance and some difficulty to 152 people, mobility indoors to 140 , shopping to 131 people, and public transport to 133 people. In initial Rasch analysis, item 1 ('ask for help from passers by') had an outfit meansquare value of 2.14, indicating that this question was answered on a sufficiently different basis from the other questions that it did not fit an underlying unidimensional construct, and was removed from the scale. 
The analysis was repeated with this item excluded, and the resulting item parameters are given in

Table 5. Category functions were none, $-2.18,-0.26,0.64$, and 1.80 logits, with each category the most probable response at some point on the scale. Person separation was 4.62 and reliability 0.96 , item separation was 5.51 and reliability 0.97 , and targeting was $+0.12 \pm 1.60$ logits, all of which are acceptable. Item 11 has an outfit of 1.71, and item 6 has an infit of 1.63, with all other items having fits within the range 0.5 to 1.5 . It should be noted that as the task questions were only asked to participants who found a goal important and difficult, the targeting value given reflects only the views of those who found the goal difficult and may thus overestimate perceived difficulty. However, even the least relevant goal (shopping) was important and of some difficulty to 131 people (79\% of the sample) such that the effect of excluding those who found the overlying goal 'not difficult' is likely to be relatively minor.

This overview analysis allows consideration of the most difficult tasks underpinning the most difficult goals for someone with Retinitis Pigmentosa, which may need to be addressed by rehabilitation. The key areas identified include orientation in difficult lighting conditions (both dim and bright light, and in indoor and outdoor conditions), avoiding obstructions (particularly outdoors), and visual search tasks such as finding products in unfamiliar shops.

Tasks that are not reported to be difficult include travelling without getting tired, travelling in familiar environments, and some aspects of using public transport. Also of note is that going up stairs is reported to be rather less difficult than walking down stairs.

$\underline{\text { Unidimensionality and subscales }}$

Having considered the unidimensionality of the scale through item fits, it is necessary to consider variance explained and contrasts in the data as further evidence of unidimensionality. The variance explained by the measures is $59.3 \%$, close to the $60 \%$ suggested as optimal. ${ }^{23}$ The raw variance explained by the items in the principal Rasch analysis (13.7\%) is less than twice the unexplained variance in the first contrast $(7.5 \%)$, showing that there is a noticeable additional dimension to the 
171 primary Rasch dimension. ${ }^{21}$ There are also five significant contrasts in the data, with the first having

172 a strength of 7.7 eigenunits. Such a lack of strict unidimensionality might be expected, since the task

173 questions cover a range of areas of activities of daily living from mobility and domestic life domains.

174 However it is relevant to consider whether the items of the overview scale can be separated into more specifically unidimensional subscales that identify constructs that might be considered as separate rehabilitation areas to address for people with Retinitis Pigmentosa. All of these tasks are difficult for those with Retinitis Pigmentosa, but which group together in similar ways?

Items loading significantly onto the first contrast of the overview scale $(5,6,25-29,36-39,42)$ were identified as answered in a different way to the underlying latent trait of the overview scale, and evaluated as a separate subscale. The items formed a subscale with poor item characteristics (separation 1.56, reliability 0.71 ), and a first contrast of 3.2 eigenunits. Therefore, the items forming the first contrast of this subscale $(28,29,27,26)$ were evaluated separately. These 4 items then made a cohesive subscale around 'Finding Products' (Table 6a, Subscale 1), with adequate scale parameters apart from slightly low item separation (Table 7). Poor item separation can be driven by insufficient respondents, or by a lack of variation in the item difficulties of the questions. ${ }^{22}$ The latter is more likely to be an issue in this instance, given that each item was applicable to 123 or more participants, but the difficulties of these items in the overview scale ranged only between +0.28 and -0.14 logits (Table 5).

The full scale was reanalysed, excluding items 1 (poor fit) and 26-29 (subscale 1). The first contrast of this analysis (6.4 eigenunits) included items $36,37,38,42,39,5,6$, and 43 loading onto it significantly. Analysis of these items separately produced a scale with a first contrast of 2.5 eigenunits, with items 5 and 6 loading significantly onto this. These were removed and the remaining items reanalysed. The resulting subscale consisted of items $36-39,42$ and 43 and made a cohesive subscale around 'Using Public Transport' (Table 6a, Subscale 2) with acceptable scale parameters (Table 7). 

(subscale 2). The resultant scale had a first contrast of 3.75 eigenunits, with items 5, 4, 6, 25 and 35 loading significantly onto it. These items made a cohesive subscale around 'Utilising Visual Information' (Table 6a, Subscale 3). Scale parameters are all acceptable (Table 7).

201 Reanalysis of the full scale, excluding the items already accounted for, resulted in a scale with a first contrast of 3.01 eigenunits, to which items 13,11, 16, 17 contributed significantly. These items made a cohesive subscale around 'Poor Light and (Inferior) Obstacles' (Table 6a, Subscale 4). Scale parameters are acceptable (Table 7) apart from targeting: the ability expressed by this group was low compared to the difficulty of the questions, reflecting that these questions were the most difficult for those with Retinitis Pigmentosa in the overview scale.

In the next iteration of the full scale analysis excluding items already used, the first contrast was 2.6 eigenunits and was loaded onto significantly by items $20,21,19$, and 22 . These items made a cohesive subscale around 'Going Up and Down' (Table 6b, Subscale 5), with acceptable scale parameters (Table 7).

The first contrast in the next iteration had a value of 2.32 eigenunits, and included items 30 and 9 (getting to places without getting tired, from the mobility outdoors and public transport goals). However, these two questions formed a poor subscale with an item separation of 0.79 and reliability of 0.38 . They were also relatively easy questions in the overview scale and thus also had poor targeting of $+4.20 \pm 6.04$. These items were therefore removed without using them in a subscale and the analysis repeated. The repeated analysis had a first contrast of 2.4 eigenunits, with items 33,34 and 41 loading significantly onto it. These items made a relatively cohesive subscale with a theme of 'Public Transport Practicalities' (Table 6b, Subscale 6). Scale parameters are acceptable, apart from slightly low item separation, again due to lack of variability in item difficulty. Similarly to Subscale 1 , each item was applicable to 124 or more participants, but the difficulties of the items in the overview scale ranged only between -0.12 and -0.69 logits (Table 5). 
Repetition of the analysis revealed a first contrast of 2.1 eigenunits, onto which items 8,7 and 14 loaded significantly. These made a cohesive subscale on the theme of 'Street Safety' (Table 6b, Subscale 7). Scale parameters are all acceptable (Table 7).

The remaining items now formed a unidimensional scale with a first contrast of 1.9 eigenunits. This scale includes items $2,3,10,12,15,18,23,24,31,32$, and 40 . The theme assigned to this subscale was ‘Orientation' (Table 6b, Subscale 8). Scale parameters are all acceptable (Table 7).

\section{Differential item functioning}

To help target rehabilitation strategies most appropriately, it is also of interest to consider whether tasks are of similar difficulty for all respondents, or are of particular difficulty for certain groups. This can be revealed by considering differential item functioning, which is assessed for different demographic characteristics in Tables 8-12.

The items that the younger participants find harder than those who are older are the items relating to orientation in poor light and peripheral obstacles, which had the highest item difficulties in the overview scale. The items that are specifically harder for the older participants were easier tasks within the overview scale, and relate to finding products and use of public transport.

Although older participants tended to have had visual loss for a longer duration (duration of visual impairment $=(0.48 \times$ age $\left.)-2.51, R^{2} 0.24, p=.000\right)$, there are differences in the items with differential item functioning when considering duration of visual loss. Those who had been visually impaired for longer found some items relating to finding products and public transport more difficult, similar to the older participants. However, those who had been visually impaired for less time found the items on fatigue specifically more difficult. These were the 'easiest' items overall in the overview scale.

In terms of gender, male participants expressed more difficulty with furniture shopping and reading traffic signs. Female participants expressed more difficulty with fatigue, as had those who had been 
visually impaired for less time. Females in the sample did have a significantly $(t(163)=2.85, p=.005)$ shorter duration of visual impairment ( $19 \pm 14$ years) than males ( $25 \pm 17$ years).

247 Mobility aid users found the most difficult outdoor tasks of orientating in poor light and avoiding inferior obstacles significantly easier than those not using mobility aids. Those using mobility aids found visual search tasks around finding products more difficult, indicating that mobility aids are not helping with such central vision tasks. There were no other items with significant differences in difficulty reported by mobility aid users and non-users. Therefore, the use of mobility aids appears to equate the difficulty of mobility in general, and makes specific mobility tasks easier than for those not using aids.

The items that are specifically difficult to those with less severe impairment due to Retinitis Pigmentosa, orientating in poor light and peripheral obstacles, reflect the difficulties that people with Retinitis Pigmentosa may initially present with in terms of night blindness and restricted peripheral field. The items of greater specific difficulty for those registered severely sight impaired, around utilising visual information and finding products, reflect how further visual field restriction and central vision reductions lead to particular difficulties with visual search and central reading tasks that are not experienced in the earlier stages. Several items that are more difficult for those registered severely sight impaired are consistent with those also seen to be more difficult for those of greater age, duration of visual impairment, and who use mobility aids. As might be expected, those registered severely sight impaired are more likely to be older $(t(163)=-4.22, p=.000)$, to have been impaired for longer $(t(163)=-4.72, p=.000)$ and to be more likely to use mobility aids (Mann Whitney U 1903, Z=-5.74, $p=.000)$.

\section{Discussion}


The overview scale produced here by assessing the tasks underpinning the most difficult goals of the

Dutch Activity Inventory for people with Retinitis Pigmentosa allow consideration of the most difficult tasks that need to be incorporated into rehabilitation programs. The most challenging tasks overall (Table 5) relate to mobility in poor and bright light both outdoors and indoors, and to avoiding peripheral obstacles outdoors. These are not unexpected activity limitations, given that the effect of Retinitis Pigmentosa on photoreceptors is such that the presenting visual impairments are usually poor scotopic vision and reduced peripheral field. The high prevalence of posterior subcapsular cataract in Retinitis Pigmentosa ${ }^{24}$ is also likely to impact on difficulty in bright light conditions when the pupil constricts. Greater difficulty with mobility in reduced illumination by people with Retinitis Pigmentosa has previously been reported in questionnaire studies, ${ }^{25}$ and that cannot easily be predicted.

To take an alternative perspective, what are the least challenging of the tasks assessed and can these suggest appropriate rehabilitation strategies? Fatigue, asking for help and travelling in familiar environments are the least challenging of these tasks underpinning difficult goals (Table 5). Given these findings are for people with largely established visual impairment (median duration of loss 16 confidence in asking for help. Tiredness was found more difficult by those who had been impaired for less time, and also by women. Those who have been visually impaired for less time are likely to have less severe visual loss, but may not yet have developed techniques or compensatory strategies for achieving tasks that those with more longstanding impairment have, or gained familiarity with 
undertaking these tasks as a visually impaired person, and it therefore takes more effort to undertake and achieve these tasks.

The use of Rasch analysis also allowed the derivation of subscales, identifying unidimensional themes that might be usefully considered as independent aspects of particular difficulty for those with Retinitis Pigmentosa to be considered in the rehabilitation process. This complements the overview analysis that addresses relative item difficulty but in a slightly less unidimensional way. The analogy is that the overview scale is like an assessment of 'maths' and the subscales identify the relative components of this overarching theme, such as 'addition', 'algebra' or 'calculus'. However, the novelty of the subscales as derived here is that the tasks are reorganised from those that are grouped together by goal (researcher driven) to those perceived as requiring similar skills by people with Retinitis Pigmentosa (patient driven).

The complexity and diversity of needs makes it necessary to adapt any rehabilitation programme, such as orientation and mobility training, to an individual client's needs. ${ }^{27}$ The most effective methods to teach the use of mobility aids, or orientation and mobility in general, are unclear with no good quality evidence currently available. ${ }^{27,28}$ There has been interest in determining whether a standardised orientation and mobility teaching protocol would have better outcomes than usual care ${ }^{29}$ but the results of the trial suggested little difference between techniques ${ }^{30}$ although the trial was hampered by trainers deviating from the standardised protocols, potentially to deliver more client centred training. The subscales derived in the present study may be useful in informing the development of training programmes, suggesting tasks that can be considered together in training as forming a unidimensional construct from the client's perspective. The subscales do appear to group together skills that are considered similar by rehabilitation specialists: for example, the subscales of 'going up and down' and 'using public transport' represent higher risk activities that would be taught after more basic skills had been acquired. 
There is also little current evidence of the value of training provided by rehabilitation specialists. ${ }^{31,32}$ The subscales outlined here could provide a potentially valuable specific tool to provide evidence of success in client-centred rehabilitation training. Clients undertaking initial training could have changes in perceived ability assessed by administration of relevant subscales pre- and post-training. Following successful initial training and review of appropriate further goals, more advanced skills could be taught and efficacy assessed using further relevant subscales.

The use of differential item functioning also allows consideration of which tasks are more specifically difficult for particular groups, which can again inform the rehabilitation needs of those with Retinitis Pigmentosa. The most difficult items overall, those around poor light and obstacle avoidance (subscale 4), are even more difficult for those in the earlier stages of the condition, namely those who are younger, who do not use mobility aids, and who are either not registered or registered only as sight impaired. Since mobility difficulties tend to increase as the extent of visual field loss increases, $^{7,26,33}$ it might have been expected that mobility tasks such as avoiding peripheral obstacles would have been more difficult for those in the later stages of the condition, who are more likely to be registered as severely sight impaired. However, it could also be argued that as the disease progresses, adjustments to approaches to mobility are made from an early stage, such that these tasks seem less of an issue than they do earlier in the disease process, and newer difficulties, such as those with visual search as the field contracts to very small levels, are relatively more difficult as they need to become adapted to. Items around visual search (subscale 1) are found more difficult by those with more profound visual loss as indicated by their registration as severely sight impaired, use of mobility aids, older age and longer duration of visual impairment. Items around utilising visual information (subscale 3) are also found more difficult by those registered severely sight impaired. These specific activity limitations are likely to relate to visual impairments which become more of an issue later in the disease process, with very limited visual fields affecting visual search, and additional progressive loss of central visual 
acuity and contrast sensitivity. Use of public transport (subscale 2 ) is found more difficult by those who are older, or have been impaired for longer. Thus, the need for rehabilitation training in the use of public transport and the use of visual search strategies may need to be reviewed over time, as these areas become more difficult over time. It is a limitation of the study that by using an online questionnaire, the only indication of severity of visual loss is registration status. It is acknowledged that not every participant will be in the most appropriate registration category for their visual function, since registration is voluntary. It would be useful in future research to be able to determine visual acuity and visual field extent in order to determine at what level of visual function these tasks become problematic.

Of particular note are the items for which differential item functioning is seen between people who do and do not use mobility aids, and also the items for which differential item functioning is not seen. People using mobility aids find many tasks no more difficult than those who do not use aids (such as those included in public transport (subscales 2 and 6), going up and down (subscale 5), and street safety (subscale 7)), and express significantly less difficulty with the most difficult tasks overall of orientation in poor light and bumping into peripheral obstacles compared to people not using mobility aids. These findings provide evidence that mobility aids do make a difference to performance. While it is anecdotally clear that mobility aids such as canes enhance mobility function, since users continue to find value in using them, evidence in previous literature for the benefits of mobility aids and training is not clear cut, and the present finding is therefore relatively novel. A Cochrane systematic review ${ }^{27}$ found no high quality evidence of the effects of orientation and mobility training for adults with low vision, and very little evidence has been found in the literature ${ }^{28}$ on methodology or effectiveness of symbol cane training. Guide dog users with Retinitis Pigmentosa have, however, been shown to walk faster and with greater ease with their dog than without ${ }^{34}$. Thus, further research to demonstrate whether and how mobility aids enhance mobility function would be beneficial. A limitation of the present study with regard to mobility aid use is that we did not distinguish between symbol cane and long cane users in our 'cane users' category. The 
use of canes and guide dogs were asked about separately, and of the 82 participants who used a mobility aid, 64 used a cane only, 4 used a dog only, and 14 used both a cane and a dog. Given the low number of people in the study using guide dogs as mobility aids, the data has considered all mobility aid users together.

\section{Conclusions}

Overall, the most difficult tasks underpinning the most difficult activity and participation goals for people with Retinitis Pigmentosa are orienting in poor light and avoiding peripheral obstacles. However, we also show that these specific tasks are relatively more difficult for those who are not registered and registered as sight impaired. They are also less difficult for those people who use mobility aids, demonstrating the benefits of such aids. Those who are registered severely sight impaired find visual search tasks relatively more difficult. Derivation of unidimensional subscales identifies groups of tasks considered similarly by people with Retinitis Pigmentosa, which can be used as an evidence base for developing and assessing orientation and mobility training protocols.

\section{Acknowledgments}

We thank the members of RP Fighting Blindness for their enthusiasm in taking part in this project.

\section{References}

1. Fahim AT, Daiger SP, Weleber RG. Retinitis Pigmentosa Overview Seattle (WA): University of Washington, Seattle: GeneReviews ${ }^{\circledR}$ [Internet]; 1993-2014 [cited 2000 Aug 4 [Updated 2013 Mar 21]]. Available at: http://www.ncbi.n/m.nih.gov/books/NBK1417/. Accessed November 18, 2014.

2. Herse P. Retinitis pigmentosa: visual function and multidisciplinary management. Clin Exp Optom 2005;88:335-50.

3. Lowe J, Drasdo N. Patients' responses to Retinitis Pigmentosa. Optom Vis Sci 1992;69:182-5. 
4. Szlyk JP, Fishman GA, Alexander KR, Revelins BI, Derlacki DJ, Anderson RJ. Relationship between difficulty in performing daily activities and clinical measures of visual function in patients with retinitis pigmentosa. Arch Ophthalmol 1997;115:53-9.

5. Geruschat DR, Turano KA, Stahl JW. Traditional measures of mobility performance and retinitis pigmentosa. Optom Vis Sci 1998;75:525-37.

6. Turano KA, Geruschat DR, Stahl JW, Massof RW. Perceived visual ability for independent mobility in persons with retinitis pigmentosa. Invest Ophthalmol Vis Sci 1999;40:865-77.

7. Szlyk JP, Seiple W, Fishman GA, Alexander KR, Grover S, Mahler CL. Perceived and actual performance of daily tasks: relationship to visual function tests in individuals with retinitis pigmentosa. Ophthalmology 2001;108:65-75.

8. Sugawara T, Hagiwara A, Hiramatsu A, Ogata K, Mitamura Y, Yamamoto S. Relationship between peripheral visual field loss and vision-related quality of life in patients with retinitis pigmentosa. Eye 2010;24:535-9.

9. Sandberg MA, Gaudio MR. Reading speed of patients with advanced retinitis pigmentosa or choroideremia. Retina 2006;26:80-8.

10. Bruijning JE, van Nispen RMA, Verstraten PFJ, van Rens GHMB. A Dutch ICF version of the Activity Inventory: Results from focus groups with visually impaired persons and experts. Ophthalmic Epidemiol 2010;17:366-77.

11. Bruijning J, van Nispen R, Knol D, van Rens G. Low Vision Rehabilitation Plans Comparing Two Intake Methods. Optom Vis Sci 2012;89:203-14

12. Bruijning JE, van Rens G, Knol D, van Nispen R. Psychometric Analyses to Improve the Dutch ICF Activity Inventory. Optom Vis Sci 2013;90:806-19

13. World Health Organization. International Classification of Functioning, Disability and Health (ICF). 2001. Available at: http://www.who.int/classifications/icf/en/. Accessed June 2, 2016.

14. Latham K, Baranian M, Timmis MA, Pardhan S. Difficulties with goals of the Dutch ICF Activity Inventory: perceptions of those with Retinitis Pigmentosa and of those who support them. Invest Ophthalmol Vis Sci 2015;56:2381-91.

15. Latham K, Baranian M, Timmis M, Pardhan S. Emotional Health of People with Visual Impairment Caused by Retinitis Pigmentosa. PLoS one 2015;10:e0145866.

16. Department of Health. Certificate of Vision Impairment: Explanatory Notes for Consultant Ophthalmologists and Hospital Eye Clinic Staff. 2013. Available at:

https://www.gov.uk/government/uploads/system/uploads/attachment data/file/213286/CVIExplanatory-notes-in-DH-template.pdf. Accessed June 2, 2016.

17. Rasch G. Probabalistic models for some intelligence and attainment tests. Chicago: Mesa Press; 1993.

18. Bond TG, Fox CM. Applying the Rasch model: fundamental measurement in the human sciences. Mahwah, New Jersey: Lawrence Erlbaum Associates; 2001.

19. Pesudovs K, Burr JM, Harley C, Elliott DB. The development, assessment and selection of questionnaires. Optom Vis Sci 2007;84:663-74.

20. Andrich DA. A rating scale formulation for ordered response categories. Psychometrika 1978;43:561-73.

21. Linacre JM. Dimensionality investigation - an example. Available at:

http://www.winsteps.com/winman/multidimensionality.htm. Accessed November 12, 2014.

22. Linacre JM. Reliability and separation of measures. Available at:

http://www.winsteps.com/winman/reliability.htm. Accessed 30 June, 2015.

23. Gothwal VK, Wright TA, Lamoureux EL, Pesudovs K. Rasch Analysis of Visual Function and Quality of Life Questionnaires. Optom Vis Sci 2009;86:1160-8

24. Fishman GA, Anderson RJ, Lourenco P. Prevalence of posterior subcapsular lens opacities in patients with retinitis pigmentosa. Brit J Ophthalmol 1985;69:263-6.

25. Smith AJ, de L'Aune W, Geruschat DR. Low vision mobility problems: Perceptions of O\&M specialists and persons with low vision. J Vis Impair Blin 1992;86:58-62. 
26. Black A, Lovie-Kitchin JE, Woods RL, Arnold N, Byrnes J, Murrish J. Mobility performance with retinitis pigmentosa. Clin Exp Optom 1997;80:1-12.

27. Virgili G, Rubin G. Orientation and mobility training for adults with low vision. Cochrane Database of Systematic Reviews 2010. Issue 5, Art. No.: CD003925. Available at:

http://www.cochrane.org/CD003925/EYES orientation-and-mobility-training-for-people-with-lowvision. Accessed June 2, 2016.

28. Ballemans J, Kempen GI, Zijlstra GR. Orientation and mobility training for partially-sighted older adults using an identification cane: a systematic review. Clin Rehabil 2011;25:880-91.

29. Zijlstra GA, van Rens GH, Scherder EJ, Brouwer DM, van der Velde J, Verstraten PF, et al. Effects and feasibility of a standardised orientation and mobility training in using an identification cane for older adults with low vision: design of a randomised controlled trial. BMC Health Serv Res 2009;9:153.

30. Ballemans J, Zijlstra GR, van Rens GH, Schouten JS, Kempen GI. Usefulness and acceptability of a standardised orientation and mobility training for partially-sighted older adults using an identification cane. BMC Health Serv Res 2012;12:1-14.

31. Binns AM, Bunce C, Dickinson C, Harper R, Tudor-Edwards R, Woodhouse M, et al. How Effective is Low Vision Service Provision? A Systematic Review. Survey of Ophthalmology 2012;57:34-65.

32. Acton JH, Molik B, Binns A, Court H, Margrain TH. Effect of rehabilitation worker input on visual function outcomes in individuals with low vision: study protocol for a randomised controlled trial. Trials 2016; 17:1-8.

33. Haymes $S A$, Guest $D$, Heyes AD, Johnston AW. Mobility of people with retinitis pigmentosa as a function of vision and psychological variables. Optom Vis Sci 1996;73:621-37.

34. Zabihaylo C, Couturier J-A, Termoz N, Prince F. Analysing the postural and gait behaviour of a person with retinitis pigmentosa travelling with a guide dog. Vision 2005 - Proceedings of the International Congress held between 4 and 7 April 2005 in London, UK. International Congress Series. 2005;1282:767-71. Available at:

http://www.sciencedirect.com/science/article/pii/S0531513105008022. Accessed June 2, 2016. 